\title{
The first development of human factors engineering requirements for application to ground task design for a NASA flight program
}

\author{
H. Charles Dischinger, Jr. \\ NASA/George C. Marshall Space Flight Center \\ Damon B. Stambolian \\ Darcy H. Miller \\ NASA/John F. Kennedy Space Center
}

\begin{abstract}
The National Aeronautics and Space Administration has long applied standards-derived human engineering requirements to the development of hardware and software for use by astronauts while in flight. The most important source of these requirements has been NASASTD-3000. While there have been several ground systems human engineering requirements documents, none has been applicable to the flight system as handled at NASA's launch facility at Kennedy Space Center. At the time of the development of previous human launch systems, there were other considerations that were deemed more important than developing worksites for ground crews; e.g., hardware development schedule and vehicle performance. However, experience with these systems has shown that failure to design for ground tasks has resulted in launch schedule delays, ground operations that are more costly than they might be, and threats to flight safety. As the Agency begins the development of new systems to return humans to the moon, the new Constellation Program is addressing this issue with a new set of human engineering requirements. Among these requirements is a subset that will apply to the design of the flight components and that is intended to assure ground crew success in vehicle assembly and maintenance tasks. These requirements address worksite design for usability and for ground crew safety.
\end{abstract}

\section{INTRODUCTION}

The National Aeronautics and Space Administration (NASA) has long employed human factors requirements for development of flight systems. NASA-STD- $3000^{1}$ was developed in the 1980s and has been applied to NASA human flight programs through a program-owned requirements document. The program chose those requirements in the standard that were applicable to programmatic missions and placed them into the requirements document, tailoring them if required to the specific application. For example, the International Space Station Program created SSP 50005 to be its human factors design specification ${ }^{2}$. Projects within the program then used the program specification as a source of requirements for the lower-tier system specifications. NASA-STD-3000 does not include human factors design requirements for ground tasks, and therefore, programs have not been required to develop human factors requirements for ground crew tasks. Ground systems have applied human factors requirements to some systems, but this application has been sporadic, and these have not been based on programmatic direction. As a result, there has been inconsistent use and interpretation. Very importantly, requirements for worksite design and ground crew safety have never been applicable to flight systems. The result has been that ground crews have had to develop complicated strategies for accomplishment of ground assembly and maintenance of flight systems. This has increased the risk of human error and added time to these tasks, both for task completion and for assessment of successful task completion (i.e., quality assurance, QA). This has resulted in much higher expense for ground processes of flight systems than would be the case if human factors had been designed in.

The Constellation Program (the execution program for the Exploration Vision) has accepted the responsibility, imposed by the NASA Administrator, to find ways to reduce ground operations costs. One of the ways the Program is doing this is through the application of human factors design requirements to flight systems. The intent is to design these systems to be more reliably and easily worked on while under NASA control.

\section{HISTORY OF HUMAN FACTORS DESIGN STANDARDS AT NASA}

NASA-STD-3000, developed as an Agency standard, was derived, in large part, from a Center-level standard developed by the Marshall Space Flight Center (MSFC) in the 1970s. The Man/System Requirements for Weightless Environments ${ }^{3}$ was created by MSFC based on experience with Skylab, which was the first NASA 
project with large interior vehicle volumes and planned tasks requiring worksite design. This document was, in turn, derived from an earlier MSFC standard, developed for ground work tasks at MSFC during Saturn Launch Vehicle assembly, the Standard Human Engineering Design Criteria ${ }^{4}$. Finally, this document was inspired by the efforts of the developers of MIL-STD-1472, which was being created at the same time ${ }^{5}$.

\section{DEVELOPMENT OF REQUIREMENTS FOR CONSTELLATION}

\section{PROCESS}

A driving goal for Exploration, as defined in the Vision for Space Exploration and in various speeches by the NASA Administrator is to reduce launch costs by reducing ground operations costs by an order of magnitude over the costs incurred by the Shuttle ${ }^{6,7}$. The Space Shuttle is the most capable space vehicle yet created, but its flight robustness comes at the cost of ground processing investment. Given the reality that NASA must survive with stable funding, there is no reasonable way to continue to fly the Shuttle, investing as much as the United States does in these flights, and to move beyond Earth Orbit at the same time, so the concept of a new launch vehicle to replace the Shuttle was introduced. This new vehicle will be simpler than the Shuttle, and it therefore will be inherently less expensive to operate. In addition, programmatic requirements have been developed to reduce ground operations costs as much as is feasible. Among these is a set of requirements included as a chapter in the programmatic human engineering requirements document, the Human Systems Integration Requirements ${ }^{8}$ (HSIR). This set comprises a chapter in the HSIR, and the requirements apply to the design of the flight systems. That is, they are applied to the vehicle to be flown and control worksite design for those human tasks that NASA or its contractors will have to perform on the vehicle, primarily at KSC.

\section{RATIONALE AND APPLICABILITY}

In general, NASA does not concern itself with processes in a contractor's facility, except where those processes affect flight performance. Thus, the Agency imposes manufacturing standards and "best-practices" direction for welding, soldering, machining, and the like. It does not generally direct a manufacturer to design worksites for hardware manufacture to accommodate a range of worker sizes and so forth. This is under the purview of the manufacturer, in the belief that manufacturing companies will develop assembly efficiencies that suit their schedules and corporate goals. The manufacturer accepts responsibility for decisions about how to put the pieces together efficiently and safely. On the other hand, assembly of launch vehicles from components delivered by these manufacturers is the responsibility of NASA. After these components (e.g., External Tanks, Solid Rocket Boosters, and Orbiters, in the Space Shuttle Program) arrive at the launch integration site
(KSC), they are owned by NASA and are no longer the responsibility of the manufacturers. Similarly, the Agency and its operations contractors will be responsible for the assembly of the launch stack for Constellation, and it is the interfaces which NASA will have to interact with that the requirements apply to.

Considerable experience with ground tasks associated with the processing of space launch systems leads to the conclusion that failure to design systems for ground crew interfaces adds to processing time and introduces risk $^{9,10}$. This is because failure to design for human tasks results in tasks that are challenging for ground crews to accomplish. Operational workarounds must be implemented at the launch assembly site. At a minimum, these workarounds add complexity by requiring more personnel than might optimally have been needed, which results in increased operational costs. More worrisome, some tasks become inherently risky, either through added complexity or by placing demands on ground personnel which result in increased error. For example, there are numerous task sites within the Space Shuttle Orbiter which provide insufficient work envelope; this often results in a worker mating connectors or driving bolts overhead, or in some other awkward position, and in the blind. In such cases, the forces required for task completion may exceed the normal range of capabilities. This results in special selection of technicians to perform the task: individuals with long reach, possibly thin body, and high shoulder, arm, and wrist strength. Specific workarounds such as this slow processing operations, while the individual capable of the task is scheduled into the workflow. Such specialized scheduling will invariably lead to delays and therefore add to costs. Moreover, the inspection of the task by QA personnel may not really be feasible, since another individual with the same anthropometric characteristics will be difficult to locate within the QA staff. It is thus possible that an improperly secured connection or insufficiently torqued bolt will result in a failure during the launch phase, when the vehicle is shaken violently. The failure could be that the connector demates, resulting in an electronics (power, computer, communication) failure. In the case of the improperly tightened bolt, the hardware could come loose, damaging or destroying it and its function, and possibly damaging other hardware nearby. Previous NASA launch system design activities have not imposed human factors engineering requirements, primarily for two reasons. The focus of the design has been on the flight performance of the vehicle: how well it accomplishes its primary objective of getting crews and payloads to orbit. Secondly, the issues mentioned above of operational costs and risks incurred were not fully appreciated. In fact, it is primarily through the Space Shuttle Program that we have learned about these issues. During Mercury, Gemini, and Apollo, the costs were simply not the driving concern; very large numbers of technicians were employed to meet the schedule of the ambitious goal of reaching the moon in the decade. Such numbers simply swamped the issues of finding anthropometrically-appropriate workers. A 
high proportion of the large costs for Shuttle ground processing is due to the number of personnel required, and this provides an opportunity for the Constellation Program: reduction of ground processing workforce will result in direct reduction of ground operations costs, an Agency objective ${ }^{11}$.

\section{DEVELOPMENT OF THE REQUIREMENTS IN THE PROGRAM}

In the summer and fall of 2006, the Agency was in the process of developing the requirements set for all aspects of the Constellation Program. The process began with the definition of missions, and performance and design requirements were identified that would support the needs, goals, and objectives associated with these missions. The missions identified included support to the International Space Station (ISS), in the form of crew changeout and supply delivery, as well as definition of the types of lunar missions to be accomplished. Both types of missions call for launch capabilities similar to those of the 1960s. While the launch rate itself will not be as high as during that decade, there are requirements for two launches within ninety minutes and for rapid assembly of the launch stack upon arrival of components at KSC. These capabilities are driven by and correlated to the launch "availability," which is a measure of ease of assembly and of the reliability of the launch system ${ }^{12}$. In support of these programmatic goals, the authors proposed that human engineering requirements be developed for ground human tasks associated with the assembly of the vehicle and any maintenance activities that might be required to assure the desired launch availability. The program readily declared that such requirements were needed to meet these goals and tasked the authors to develop them. One of us (DS) had considerable experience in identifying ground task requirements for flight systems, and the other two had experience with development of requirements for spaceflight and for ground systems ${ }^{9}$.

\section{STRUCTURE AND NATURE OF THE REQUIREMENTS}

The primary objective of the team was to develop a basic set of requirements that could provide projects within the program a starting point from which to derive a set of requirements appropriate to that project. This was driven by two considerations: there was little time to invest in development of a complete set, and the philosophical approach was that there should be latitude for interpretation and trading of design requirements against other considerations. The requirements were not part of the NASA flight human factors requirements set found in NASA-STD-3000, as previously mentioned. This made the addition to the HSIR a rush job, and examples were needed. The earlier NASA standards documents which did include ground design requirements were somewhat dated, but more importantly, they focused on manufacturing processes and facility design to a large extent. This set of requirements was conceived and commissioned to influence design for vehicle assembly and those maintenance tasks to be performed only at KSC, as preciously mentioned. MIL-STD-1472F was considered to be a more up-to-date starting point, and it was in use at KSC for design of ground systems. It was culled for requirements which might be applicable or adaptable to space flight systems. The other consideration, that there be latitude for tailoring for specific projects, enabled selection of generic requirements. In general, human factors engineering requirements for spaceflight application are "hard" requirements, meaning they must be strictly adhered to. This is because many tasks during a mission must be done correctly "first time, every time." Task error can have direct and immediate impact on crew safety and mission success. On the other hand, ground task design can reasonably be considered a tradable commodity, when considering overall vehicle performance. Thus, if the imposition of human factors engineering requirements for ground tasks resulted in significant increases in weight, the sense among the team was that projects should be allowed some leeway in the imposition of these requirements, if suitable workarounds can be planned that could be demonstrated to result in a safe design. Such tailoring of requirements is achieved through programmatic concurrence, to assure the overall program objectives of launch availability and mission capability are met. It was with this thinking that the selection of a small generic set of requirements was made. The process was fairly quick, from a requirements development standpoint, because this approach did not demand that a great deal of supporting data be provided. The requirements addressed primarily the basic demands of worksite development: reach envelopes, body and work envelopes, tool envelopes, and visual envelopes. In addition to these, some specific requirements were added, based on experience from ground processing personnel at KSC.

\section{EXAMPLES}

The Constellation Program adopted a policy for all requirements documents that each requirement be matched to a rationale statement and a verification requirement. The rationale shows up in the document immediately under the requirement and is intended to explain the need for the requirement and, if necessary, the thought process behind it, to aid in interpretation. The verification requirement states how the design requirement is to be verified; this provides additional information to the hardware developer of the intent and helps assure the requirement is well-written.

\author{
Example of a general requirement, rationale, and \\ verification requirement: 3.9.6.7 Work Envelope \\ Volumes [HS10002] The system shall provide work \\ envelope volumes needed to perform \\ corrective and preventive maintenance tasks, as well as \\ assembly and other launch site \\ processing tasks. \\ Rationale: The flight system components/subsystems
}


(Ares I stages, Orion Service Module [SM] and Crew Module [CM], e.g.,) must be assembled by the ground crew with sufficient work envelope to accomplish tasks. Many of these tasks will constitute mating of components (bolts, connectors, etc.) across the interface between Elements (Ares I 1st: 2nd stage, e.g.,) or between systems (Ares I: Orion). These envelopes will therefore be identified by Vehicle-level task analyses and documented in Interface Control Documents (ICDs). Corrective and preventive maintenance tasks that are accomplished fully within one Element may be analyzed at the Element level. Guidelines for envelope definition are found in FAA-HF-STD- 001 Section 14.1. Sufficient envelope is defined by task analyst using this document and based on anthropometric requirements and task definition. The envelope definition will be concurred with by Level II.

4.9.6.7 Work Envelope Volumes

[HS10002V] Assembly and maintenance work envelope volumes shall be verified by analysis. The analysis shall consist of task and worksite analysis. The Vehicle Assembly Task Analysis and the Vehicle Maintenance Task Analysis shall be applied to determine task assumptions and constraints (e.g., SCAPE suit), and the worksite analysis shall account for constraints. Analysis shall account for the anthropometric range as applicable, the task, and the environmental constraints. The verification shall be considered successful when the analysis shows that the tasks have the needed work envelope volumes.

Rationale: No further Rationale required.

Example of a requirement that is based on lessonslearned from experience at KSC: 3.9.7.7.7 Protrusion Label/Support [HS10043] The system shall design all accessible protrusions that could be inadvertently used as handles, steps, hand rails, or mobility aids either to support the weight of personnel or clearly labeled as keep-out zone.

Rationale: Historical experience with Shuttle and Station has shown that it is important to make it clear which parts of a vehicle may not be used as handles, steps, or handrails so that as ground and flight crews move around the vehicle they do not inadvertently damage delicate portions. Preference should be given to designing to support in areas where ground and flight crews will travel frequently.

4.9.7.7.7 Protrusion Label/Support

[HS10043V] Protrusions that could be used as handles, steps, or hand rails shall be verified by analysis. The analysis shall determine which protrusions could be inadvertently used for handles, steps, hand rails, or mobility aids. The verification shall be considered successful when the analysis shows that the identified protrusions accessible to the ground and flight crews can support the weight of personnel or are clearly labeled as a Keep Out Zone.

Rationale: No further Rationale required.

\section{ISSUES AND CHALLENGES}

Some requirements have presented challenges, but not all of these have been technical. Verifiability has been a significant concern. For example, the anthropometry requirement, as originally stated, said that " $5^{\text {th }}$ to $95^{\text {th }}$ percentile" workers would be accommodated. This is a common range of workers to be designed for, but, as anyone who has tried to apply it will understand, its use is not straightforward. The question, familiar to all worksite designers, is, "Which anthropometric dimension needs to fall within this range?" Often, designers are encouraged to select those dimensions which are most critical for the task being designed for. In an attempt to simplify the requirement, the authors added the phrase, "for stature," but even this is unsatisfactory. Moreover, the requirement did not specify a database on which to draw in order to develop worksite design. It was our intent to have hardware developers (manufacturers) inform the Agency how they had arrived at the anthropometries they used in the design. This was considered unsatisfactory, for two perfectly reasonable reasons: it required the developer to expend time and effort (and hence, money) to decide on a database, and different developers could decide to use different databases. This could result in inconsistency of design and potential worksite design problems at the interfaces between hardware delivered by different developers. Thus, the Upper (second) Stage of the Ares I launch vehicle could select a different dataset than the Orion crewed vehicle, and integration problems could arise when these components are mated at Kennedy Space Center. The most recent version of the HSIR still does not specify the applicable database, but the author's proposal is to simply use the human modeling tool as the standard for anthropometry. The Constellation Program has selected a CAD and simulation system which includes a human model for design and analysis, and this model is scalable; the position is to use the anthropometries designed into the model. The verification would then be straightforward: use of the human model to demonstrate task feasibility for the range inherent in the model would allow verification closure. This would support one of the basic concepts behind inclusion of anthropometry in design considerations: it gets the worksite design "in the ballpark" for the workers who must perform tasks. All qualified worksite designers recognize that no design will accommodate all potential workers; the intent of the imposition of such a requirement is to assure that worker sizes have been considered in the design, with the understanding that some workers will not be able to perform some tasks.

Another requirement which has been difficult to establish is one calling for a defined tool set ([HS10028] The system shall be assembled and maintained using only those tools identified in the Launch Site Task Tool List (TBD-006-050).). While not uniquely a human factors design requirement (it supports logistics planning, as well), it is intended to allow a worksite designer to understand the tools that might be selected for a task, 
enabling assessment of tool envelopes. It was created with the idea that a tool list already exists for Shuttle, and that this could be transferred to Constellation. A complete list has been difficult to acquire, however. This appears to be related to contractual issues; the KSC contractor which manages tools is not obligated to provide the list to Constellation. While this should ultimately be resolvable, the designers are currently without a tool set.

\section{CONCLUSION}

One of the goals of the Exploration Vision is a reduction of costs associated with launch processing. The primary goals of Exploration are, of course, discovery of the nature of other bodies in the solar system and establishing permanent human presence there. Improving ground processing is thus not a primary goal, but it is an enabling one. The idea is that NASA budgets are unlikely to increase in the foreseeable future, and the Agency must learn to reduce ground operations costs in order to have a greater proportion of its budget go to space applications. One of the paths Constellation is using to achieve this goal is the application of human factors requirements to the design of worksites for launch vehicle assembly and maintenance. These requirements have gone into programmatic documentation as additions to the flight crew human factors requirements in the HSIR and are applicable to the flight systems only.

\section{REFERENCES}

1. 1989. Man-System Integration Standard. Vol.1, Rev. A. NASA-STD-3000.

2. 1994. International Space Station Crew Integration Standard, Rev. A SSP 50005.

3. 1974. Man/System Requirements for Weightless Environments, Rev. A. MSFC-STD-512A.

4. 1966. Standard Human Engineering Design Criteria. MSFC-STD-267A

5. 1968 and ff. Department of Defense Human Engineering Design Criteria for Military Systems, Equipment, and Facilities. MIL-STD-1472 (initial, with revisions through $F$ ).

6. 2004. Speech by the President, 14 January and NASA document The Vision For Space Exploration, February.

7. See remarks to National Space Club, 02.07.07, at http://www.nasa.gov/news/speeches/admin/mg spe ech collection archive 2.html

8. 2006. Human Systems Integration Requirements. CxP 70024.

9. 2004. D. B. Stambolian and T. Greenfield. Spaceport 1-G human factors for optimal space transportation system design.

10. 2004. D. Stambolian and T. Foley. NASA Lessons Learned 1801.http://nen.nasa.gov/portal/site/lis/index.jsp?epicontent=LLKN DOCUMENT VIEWER\&llknDocUrl=http\%3A\%2F \%2Fnen.nasa.gov\%2Fllis content\%2Fimported content\%2Fless on 1801.html\&llknDocTitle=Lessons\%20Learned\%20Entry:\%201 $\underline{801}$

11. 2008. Exploration Needs, Goals, and Objectives Document. ESMD-ENGO-01.08 Rev -

12. 2007. Constellation Architecture Requirements Document. CxP 70000.

\section{CONTACT}

Charles Dischinger

EV82/Vehicle Engineering and Integration Division MSFC, AL 35812

charles.dischinger@nasa.gov 